\title{
Elasto-kinematics design of an innovative composite material suspension system
}

\author{
Shuang Xu, Alessandro Ferraris, Andrea Giancarlo Airale, and Massimiliana Carello \\ Mechanical and Aerospace Engineering Department, Politecnico di Torino, C.so Duca degli Abruzzi 24 Turin, \\ 10129, Italy \\ Correspondence to: Shuang Xu (shuang.xu@polito.it)
}

Received: 15 July 2016 - Revised: 26 January 2017 - Accepted: 6 February 2017 - Published: 24 February 2017

\begin{abstract}
In this paper, a lightweight suspension system for small urban personal transportation vehicle is presented. A CFRP (Carbon fiber reinforce polymer) beam spring has been used to efficiently integrate the functions of suspension control arm and anti-roll bar. Composites materials were chosen to tailor the required behavior of the beam spring and to reduce the weight. Furthermore, larger space for engine compartment has been provided thanks to the compact arrangement of beam suspension components. This suspension could be installed on electric/hybrid vehicles and conventional automobiles.
\end{abstract}

\section{Introduction}

The suspension system is very important in an automobile, since it directly affects the handling performance and the ride comfort. All the driving/braking force and lateral force during cornering are transferred to the car body from the ground though the suspension system.

Weight reduction is an important task in the current trend of automobile development. Aluminum alloys were commonly used to substitute steel, which can obtain a weight reduction up to $30 \%$ (Fuganti and Cupitò, 2000). However, using aluminum for structural components such as coil springs and anti-roll bars can be very difficult for effectively reducing weight while keeping the same reliability. By introducing composite materials into lightweight design ${ }^{1}$, engineers also have the possibility to improve vehicle dynamic performance. The usage of CFRP has been usually associated with high-end racing cars for building their body shell and chassis. For example, Formula 1 cars have achieved around $80 \%$ of its components made with composite material (Kulshreshtha, 2002).

Although not widely used, composite leaf springs can be found in automotive sector and in these cases the composite leaf spring has the same performance in terms of component

\footnotetext{
${ }^{1}$ More than $50 \%$ mass can be reduced using composite material (Beardmore, 1986).
}

stiffness with an increase of durability as much by five times and weight reduction of $65 \%$ (Wood, 2014). The composite leaf spring presented in this paper, is a highly deformable "beam component" made with CFRP integrating the function of spring, anti-roll bar and control arm. The proposed design saves space, system weight and complexity.

Due to the architecture chosen, the CFRP works as a complaint mechanism (CM; Hao et al., 2016), which has certain stiffness under loading during in working condition. In this research, the CFRP beam spring sustains the load to support the vehicle, while the deformation is far beyond the linear range. For this reasons particular attention has been given to manufacturing process and the simulation modeling.

\section{Suspension topology}

A front wheel driven $\left(\mathrm{FWD}^{2}\right)$ vehicle has been chosen as a case study, which is designed to use McPherson strut suspension on the front axis and SLA suspension on the rear axis. That is the most competitive combination for space saving of power train and improved lateral performance of the vehicle

The conventional McPherson suspension is well known for its simple design, compact space as well as the large shock absorber and the heavy "banana shape" lower control

\footnotetext{
${ }^{2}$ FWD: Front wheel drive, in this case, the engine is also mounted on the front axis.
} 

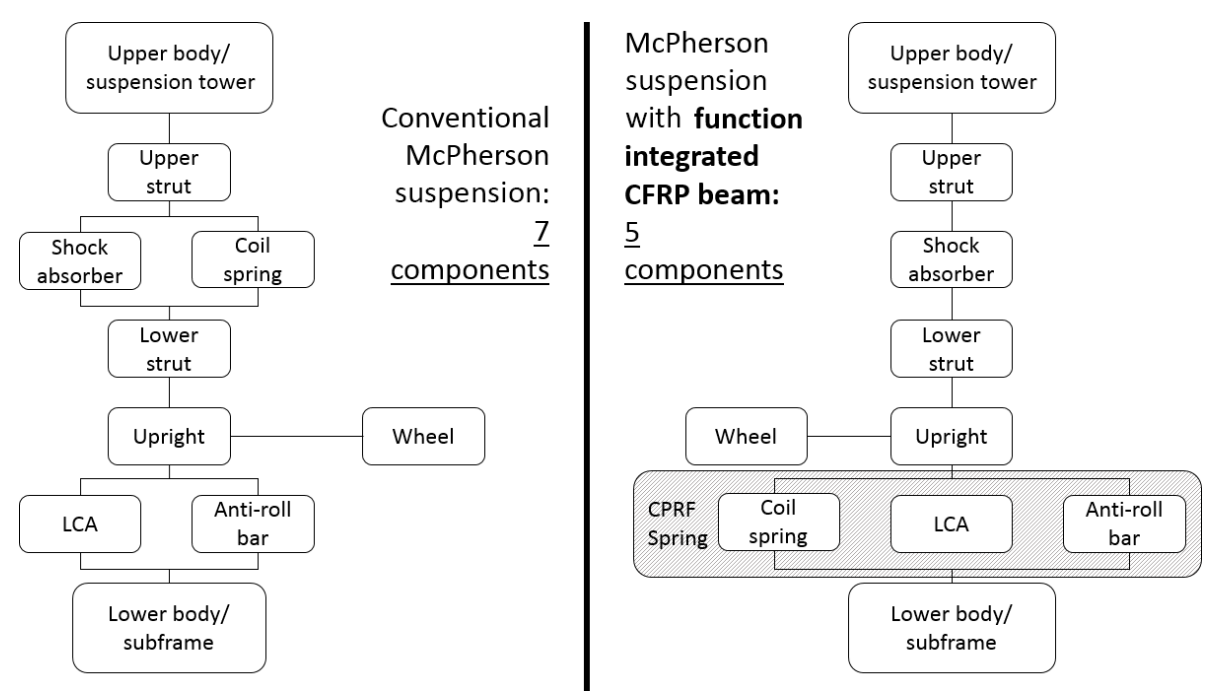

Figure 1. Topology comparison. (Upper/lower body and wheel are not count for suspension components).

arm. Since no upper control arm is present, the lateral space is larger compared to SLA suspension, which is the main reason for front mounted engine arrangement. It usually has the following components:

- Wheel hub

- Upright

- Lower control arm*

- Shock absorber (strut)

- Coil spring*

- Antiroll bar*

- Tie rod

In the purposed architecture, components marked with "**" are made redundant by integrating its function and substituted by a single CFRP beam spring as a shaded block shown in Fig. 1, the rear SLA suspension topology is similar but with an additional aluminum upper control arm.

Benefits of using a beam suspension solution are that additional space can be available with no spring (and its seat) presented and the strut can be placed closer to the wheel assembly. This can also provide better handling with a small scrub radius at relative high speed (Milliken and Milliken, 1995).

The rear suspension cannot have the same level of space reduction, but the weight is reduced by component and function integration.

\subsection{Beam spring functionality}

The most common application of CFRP is weight reduction of the chassis frame and body panels while keeping the same durability. In this application, CFRP is used to create a deformable beam spring to substitute the suspension lower control arm, which is virtually divided into three sections by the bushing mounting point on the left and right (Fig. 2).

During the parallel wheel travel (wheels of the same axis on both side moves in the same direction vertically), the beam deforms like a bow, functioning like a normal coil spring. When one wheel is moving vertically in the opposite direction of the other wheel (known as the opposite wheel travel) the beam deforms into an "S" shape, working like an anti-roll bar.

To achieve the desired vehicle dynamics performance, the stiffness of the beam under parallel wheel travel and opposite wheel travel are achieved by the correct number of the ply and the stacking sequence that is used during manufacturing of CFRP beam spring.

\subsection{Metallic components}

Using components such as upper control arm of rear SLA suspension and some other pieces made of metal (mostly aluminum alloy) has several reasons. First of all, to simplify the manufacturing process for non-definite components. Being a prototype vehicle, it is important to keep some design space and make it possible to implement some modifications of the components. Carbon fiber components are optimal when considering weight reduction, but once they have been built, it is very difficult to modify.

Besides, metallic component design procedure is relatively simple; for carbon reinforced plastics, instead, the static and dynamic loading design is difficult due to its anisotropic structure. A precise finite element analysis for such components can be very complex and unreliable. For these reasons, using the metallic components in prototyping phase is more reasonable. 


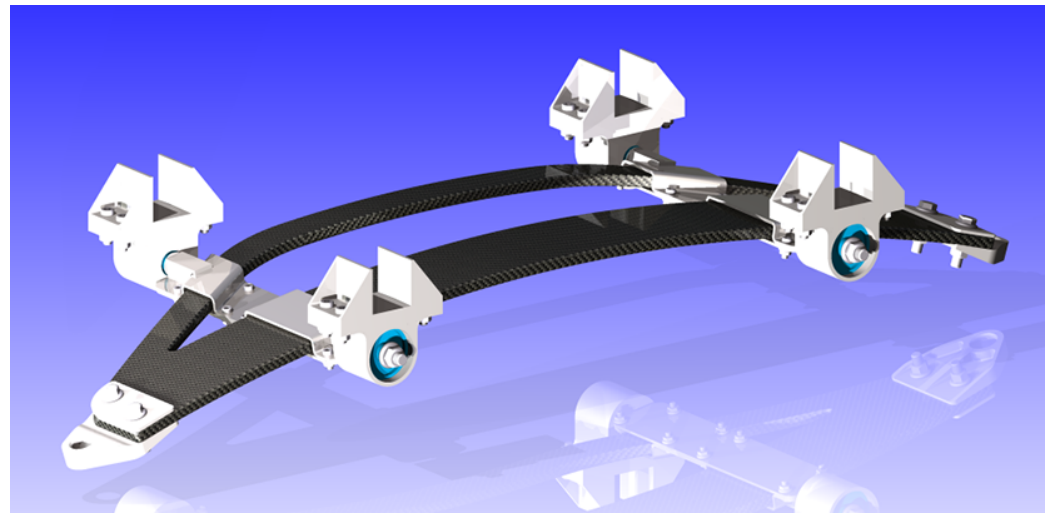

Figure 2. Unloaded front CFRP beam.

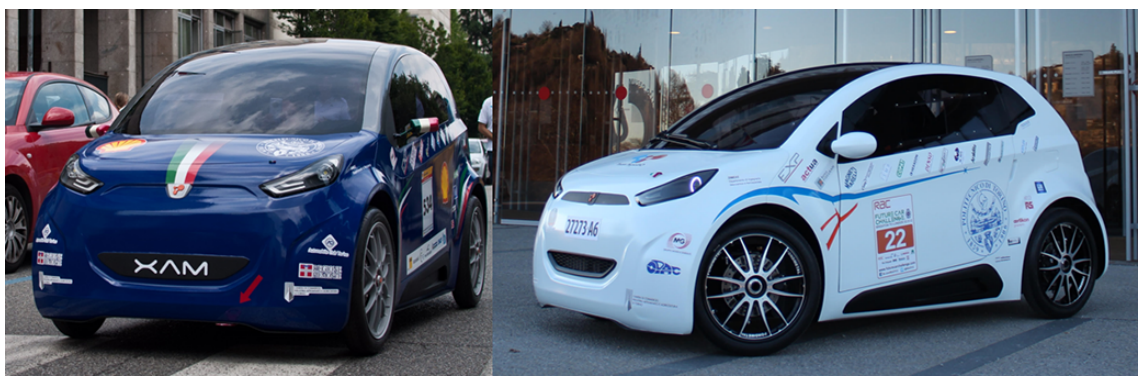

Figure 3. XAM (left), XAM 2.0 (right).

Due to this, nearly all the other components apart from the CFRP beam have been made with machined aluminum alloy. For future mass production, some of those components can be replaced with stamped aluminum sheets or hollow casted components with optimized structure design, so further weight reduction could be achieved (European Aluminum Association, 2013).

\section{Suspension modeling method}

The entire development of the prototype virtual model has been performed using MSC ADAMS/Car and Altair Radioss. The multi-body dynamic model has been made in ADAMS/Car in order to achieve a good precision on multibody simulation and modeling. A reference suspension for setting performance targets has been set-up with conventional suspensions (with coil springs) as well.

Altair Radioss has been used to correlate the material properties and the design parameters. To verify the stiffness with respect to design target after the prototyping of the beam spring, a simple test bench has been used.

The modeling of the CFRP beam suspension has been divided into 4 steps:

- Reference suspension modeling

- Rough prediction for beam spring characteristic using "Non-linear" beam tool in ADAMS
- Finite Element Method simulation and correlation with physical experiments

- "Non-linear" beam calibration with reference to the FEM results

Since it is complicated to start with CFRP beams, a "reference suspension model" with conventional suspensions has been defined in the beginning. MSC ADAMS is the most commonly used MBD simulation environment in the industry, two previous award-winning vehicles (Fig. 3) with conventional suspensions are designed successfully in the past. The experiment data on the test track is well correlated with the simulation results (Carello et al., 2014, 2012).

The reference suspension has been defined to have $\pm 70 \mathrm{~mm}$ wheel travel, and an understeering behavior has been chosen to ensure safety. Consequently, the front suspension has a $-0.4^{\circ}$ of camber angle variation at maximum stroke and a toe-out of $0.7^{\circ}$ when the coil spring is fully compressed.

Due to the rear suspension design for beam spring, no significant toe variation has been set. The camber variation for the reference rear suspension has been set to $-2.1^{\circ}$.

Beam suspension from geometric and kinematic point of view is identical for the two suspension models (reference and beam suspension models) as shown in Fig. 4. 


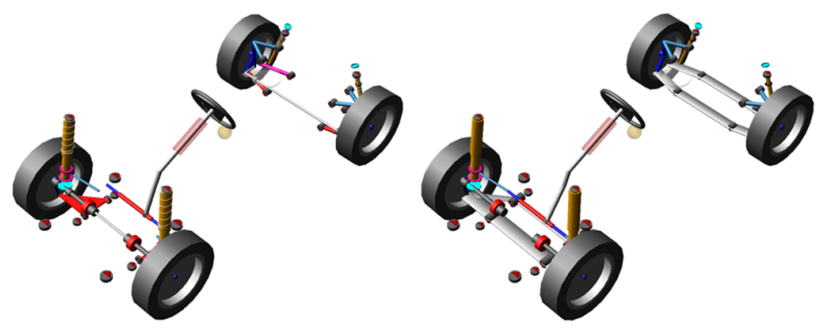

Figure 4. MBD full vehicle model, reference-handling model (left), BEAM Spring suspension vehicle prototype (right).

\subsection{Non-linear CFRP beam}

In this particular project, the beam has to be mounted transversely rather than longitudinally. The study shows in order to maximize the limit for elastic strain energy storage, the beam should be designed to have a tapered shape for vertical loading along the length (Yu and Kim, 1988). Still, a design with constant thickness along the length has been chosen to balance cost and performance, also because the space required for mounting the tapered beam is not available, and it is very difficult to prototype.

The beams have been designed to have two different shapes for front and rear axle. The beam on the front is designed to have an isosceles trapezium shape to have higher longitudinal stiffness considering driving axle may sustain heavier load on the longitudinal direction during acceleration. Another reason is that the mounting system became more reliable for constraining the beam movements during driving condition as shown in Fig. 5 for front suspension beam and its mounting design. The CFRP beam has the shape of a bow to have a higher constructional strength and curved to have correct preload.

At the end of both sides, two plates are mounted by thread fasteners, making a "sandwich structure". The beam is drilled through to let the thread fastener pass. On the lower plate, there is another hole left for the spherical joint, which is further connected with upright.

The bushing housings have been milled from a block of 7075 aluminum alloy. To prevent potential damage on the fiber beam (due to high-localized stresses) a layer of elastomer has been inserted between the beam and the steel plate.

On the rear axle, the geometry of the beam is different from the front and its shape is similar to a simple rectangular plate with curvature. The curved shape is to ensure the necessary preload when the vehicle is assembled and to permit the right vertical wheel travel same as front.

Comparing with the front McPherson suspension (shock absorber mounted between the upright and the top mount on the chassis), the rear SLA suspension shock absorber is usually mounted on the lower suspension control arm.

In Fig. 6, on the metal plate for mounting the upright, apart from the two coaxial hole (drilled to fix the upright), there is

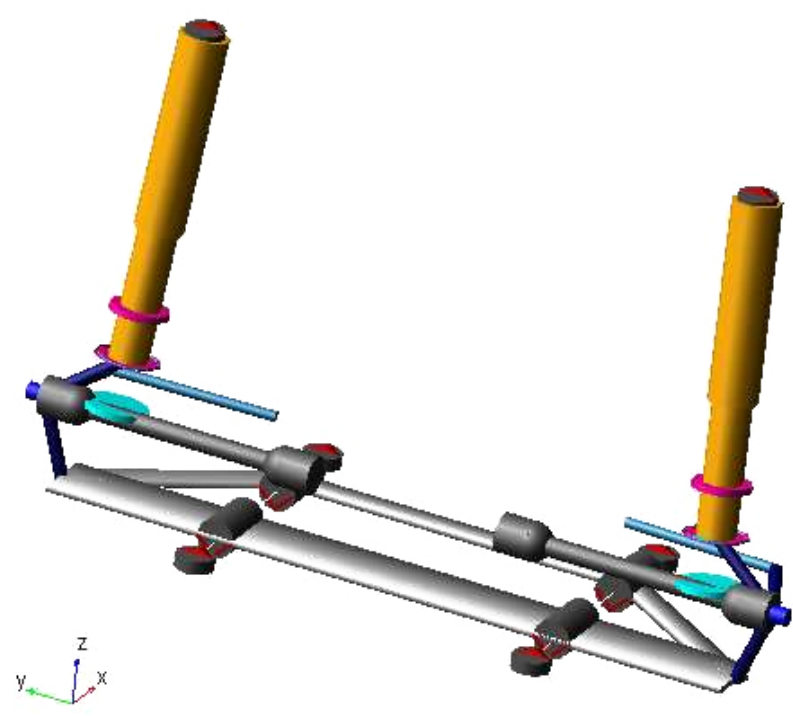

Figure 5. Front beam suspension in ADAMS/Car.

another small hole drilled on the vertical surface, for mounting the shock absorber, because drilling any more holes on the CFRP beam is not recommended for its reliability. The rear suspension is chosen to use a " $\mathrm{H}$ arm" topology ${ }^{3}$ to eliminate the need for another linkage for toe control. The side effect is that the toe variation during suspension stroke is limited.

To recreate the performance of the beam suspension, the tool "non-linear beam" in ADAMS/Car is used, which is shown in Fig. 4. The non-linear beam is defined as a flexible body made with several connected deformable segments, which is an ideal component to present the behavior of beam spring in multi-body dynamics model.

\subsection{Parametrization of beam spring}

Suspension geometry has been defined using the reference model to reach the performance target. To reach the same performance after substitution with the beam, several tuning and modification of the model may be necessary. As the beam is relatively deformable compared to the rigid control arm in conventional solution, kinematic performance for beam suspension has some variance using the same geometry from reference model.

Since CFRP beam springs have been used to substitute the lower control arm, the hardpoints of the reference suspension need to be recreated in the CFRP beam spring. Other characteristics such as the length, thickness, cross-section shape and material properties used are very important for calculating the stiffness, damping and weight during simulation with the "non-linear beam" tool for CFRP beam.

\footnotetext{
${ }^{3}$ Such as the rear suspension of Gunnell (2008) Ford Thunderbird (1992-1996).
} 


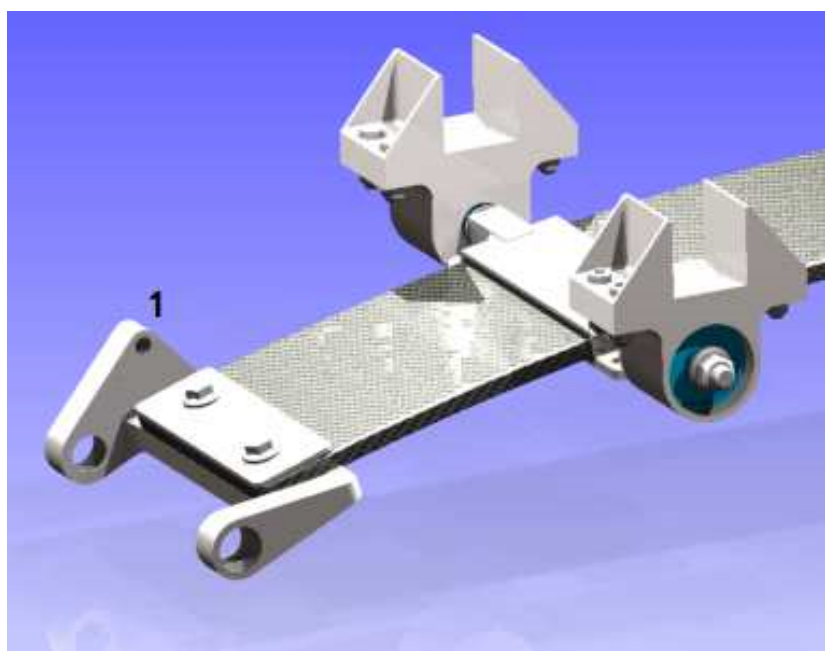

Figure 6. Drilled hole for mounting the shock absorber on rear beam (1).

To have the expected stiffness for the beam according to the performance design is very difficult, and for the FEM analysis, an initial geometry for the beam is required. In order to integrate the functionality of spring and anti-roll bar together in the single beam spring, it is difficult to obtain the desired result by manual "trial and error", considering high time consuming computational FEM simulations.

MBD simulation with DoE has been applied to obtain the correct design parameters, and the original MBD model was improved as the DoE needs parametrized model.

The beam spring has been modeled with simple parameters considering the cross section width and thickness (beam spring length is fixed by the overall design of the track width). During the development, it was intended to avoid cross section variation along the lateral direction. Variable thickness of the beam may lead to stress concentration (which increases the probability of delamination) during the deformation and production complexity will be increased as well.

It needs to be pointed out that in ADAMS/Car system, the geometry of the beam cannot be effectively controlled by the "parameter variable" ${ }^{4}$, and so it is necessary to modify the model in code level.

Once the material properties ${ }^{5}$ of CFRP are determined through experimental test, the only aspect could be modified in the design process is the moment of inertia (the crosssection). The non-linear beam component in MBD model reads the moment of inertia calculated by the parameter vari-

\footnotetext{
${ }^{4}$ The geometry can be controlled normally in the environment of template, but the simulation can only be done in the normal environment.

${ }^{5}$ The properties used in MBD model include: elastic modulus, shear modulus, Poisson ratio and density. Stacking sequence of CFRP beam spring is designed in FEM model.
}

ables using simple formulas, which can be modified to create DoE iterations.

In order to perform the calculation for moment of inertia automatically during DoE iterations, additional scripts are necessary.

Front suspension has a large slot in the center of the beam component (giving space for electric motor and other components), which is modeled as two separate beam attached with each other at the beam ending (as shown in Fig. 2). The rear beam is also divided into two separate part to achieve $\mathrm{H}$-arm structure on rear suspension. In such configuration, the beam components in the model are named as:

- Front suspension front beam (beam_front_nrl/nrr/nrs ${ }^{6}$ _lca_front)

- Front suspension rear beam (beam_front_nrl/nrr/nrs_lca_rear)

- Rear suspension front beam (beam_rear_nrl/nrr/nrs ${ }^{7}$ _lca_front)

- Rear suspension rear beam (beam_rear_nrl/nrr/nrs_lca_rear)

\subsection{DoE script preparation}

There are 3 different DoE scripts for this model:

- Generate parameter variables

- Modify beam component force calculation formula

- Create target measurement reading function

Nonlinear beam elements in a single beam are sharing the same geometry properties ${ }^{8}$ (thickness and width). Even though user can modify the thickness and width by click on different beam segment (beam elements), the beam will act as a single component just using the last input value user configured in any individual beam segment

When writing the script, it is mandatory to use "Adams/view Command", which is based on concept of "object-variable" and every variable belongs to its parent component. To make the variable accessible to DoE environment, users can choose any segment of a beam component, and reconfigure the variable of "height" (stands for thickness) and "width" into "parameter variables".

\footnotetext{
${ }^{6}$ nrl: nonlinear beam left; nrr: nonlinear beam right; nrs: nonlinear beam single.

${ }^{7} \mathrm{nrl}$ : nonlinear beam left; nrr: nonlinear beam right; nrs: nonlinear beam single.

${ }^{8}$ In Adams environment, properties of an object are called "VARIABLE", more like the "dependent variable" in general mathematic formula, its value is determined by other input values. " $\mathrm{Pa}$ rameter variables" are user-defined additional "parameters", more like the "independent variables", which give input values to the system.
} 
It needs to be pointed out that "variables" in MBD model cannot be modified DoE iteration, but "parameter variables" can be accessed easily by user using graphic interface or command line same as DoE functions. Eight individual parameter variable were created in total (two for each beam component, four beams in total). Changing the variables into parameter variables makes the beam lose the original source of properties, the force calculation is no longer valid. The force calculating functions need to be manually redirected to the new parameter variables that have been modified.

The functions of beam component forces are:

$$
\begin{aligned}
& \text { - Ixx } \\
& \text { - Iyy } \\
& \text { - Izz } \\
& \text { - Y_shear_area_ratio } \\
& \text { - Z_shear_area_ratio } \\
& \text { - Youngs_modulus } \\
& \text { - Shear_modulus } \\
& \text { - Area_of_cross_section } \\
& \text { - Damping_ratio }
\end{aligned}
$$

The above functions can be modified using Adams/view Command.

The final step for the script preparation is to create "DoE design objective" using the function builder. For this research, the beam spring stiffness under different loading cases (parallel wheel travel/opposite wheel travel) is needed and is calculated using simple "force-displacement" function.

Another function is defined as an additional indicator for beam spring stiffness: the stiffness linearity. As the experiment results show the CFRP beam spring has a certain nonperfect linear stiffness behavior. It tends to increase its stiffness when the displacement is increasing. The minimal value of stiffness appears when the beam is not loaded, similar to a uniform cubic function passing through origin. DoE shows the results only when an objective function is defined, so there is no way to observe the non-linearity without a function. The non-linearity function is very simple in this case, the function calculates the ratio $\left(r_{\mathrm{nl}}=k_{\mathrm{fs}} / k_{\mathrm{hs}}\right)$ between the mean stiffness within $1 / 2$ the total wheel displacement $\left(k_{\mathrm{hs}}: \pm 35 \mathrm{~mm}\right)$ and the stiffness at the maximum stroke $\left(k_{\mathrm{fs}}\right.$ : $\pm 70 \mathrm{~mm}$ ).

\begin{tabular}{|c|c|c|}
\hline Property & Unit & Value \\
\hline Loaded weight at SD A* & $\mathrm{kg}$ & 785 \\
\hline Weight repartition (front/rear) & - & $45 / 55$ \\
\hline Maximum loaded weight & $\mathrm{kg}$ & 870 \\
\hline Wheel base/ front track/rear track & $\mathrm{mm}$ & $1912 / 1170 / 1170$ \\
\hline Ground clearance at SD A & $\mathrm{mm}$ & 140 \\
\hline$H_{\mathrm{cg}}$ at $\mathrm{SD} \mathrm{A}$ & $\mathrm{mm}$ & 350 \\
\hline Suspension stroke & $\mathrm{mm}$ & \pm 70 \\
\hline Minimum ground clearance at SD A & $\mathrm{mm}$ & 120 \\
\hline Tire & - & 95/80R16 \\
\hline Powertrain layout & - & $\mathrm{FF}$ \\
\hline
\end{tabular}

DoE simulation has to use only one simulation script for every iteration. In this case, the script need to perform parallel wheel travel and opposite wheel travel simulation in a single simulation run. The DoE script is written to perform first parallel wheel travel, then return the wheel back to its original position, at last the suspension will be asked to perform an opposite wheel travel.
Table 1. General set-up of vehicle model.

* One driver $(75 \mathrm{~kg})$ and one luggage $(10 \mathrm{~kg})$ are included.

The design objective functions are written as "time dependent" functions. For example, wheel center force $\left(F_{\mathrm{CW}}\right)$ for calculating the $k_{\mathrm{hs}}$ is read in four different moments using Adams/View function "VALAT (array,array,REAL)"”

- FCW at $-35 \mathrm{~mm}$ for parallel wheel travel ${ }^{10}$

- FCW at $+35 \mathrm{~mm}$ for parallel wheel travel

- FCW at $-35 \mathrm{~mm}$ for opposite wheel travel

- FCW at $+35 \mathrm{~mm}$ for opposite wheel travel

The other functions are written in the same way as for $k_{\mathrm{fs}}$ and $r_{\mathrm{n} l}$.

\section{Simulation results}

The research is processed through reference model study to the beam suspension mode; the general set-up of the vehicle model is shown in Table 1. The kinematic characteristics and dynamic behavior has been defined through the simulation trails referred with specific performance targets for riding comfort and safety

DoE simulation is performed only to identify the geometry size of beam components in this article. The kinematic simulation is geometry-dependent, while tuning is based on suspension performance targets and CAD design space. Dynamic simulation is more component-dependent, as the stiffness and damping of elastic components can significantly change the dynamic response.

\footnotetext{
${ }^{9}$ The function VALAT is a linear interpolation for arrays, the measurement results are arrays for example.

${ }^{10}$ Adams/View function is written as: VALAT (ANALYSIS.left_hub_forces.TIME,ANALYSIS.left_hub_forces.normal, TIME). "TIME" is the real number for the moment when wheel hub reaches $-35 \mathrm{~mm}$ wheel travel.
} 


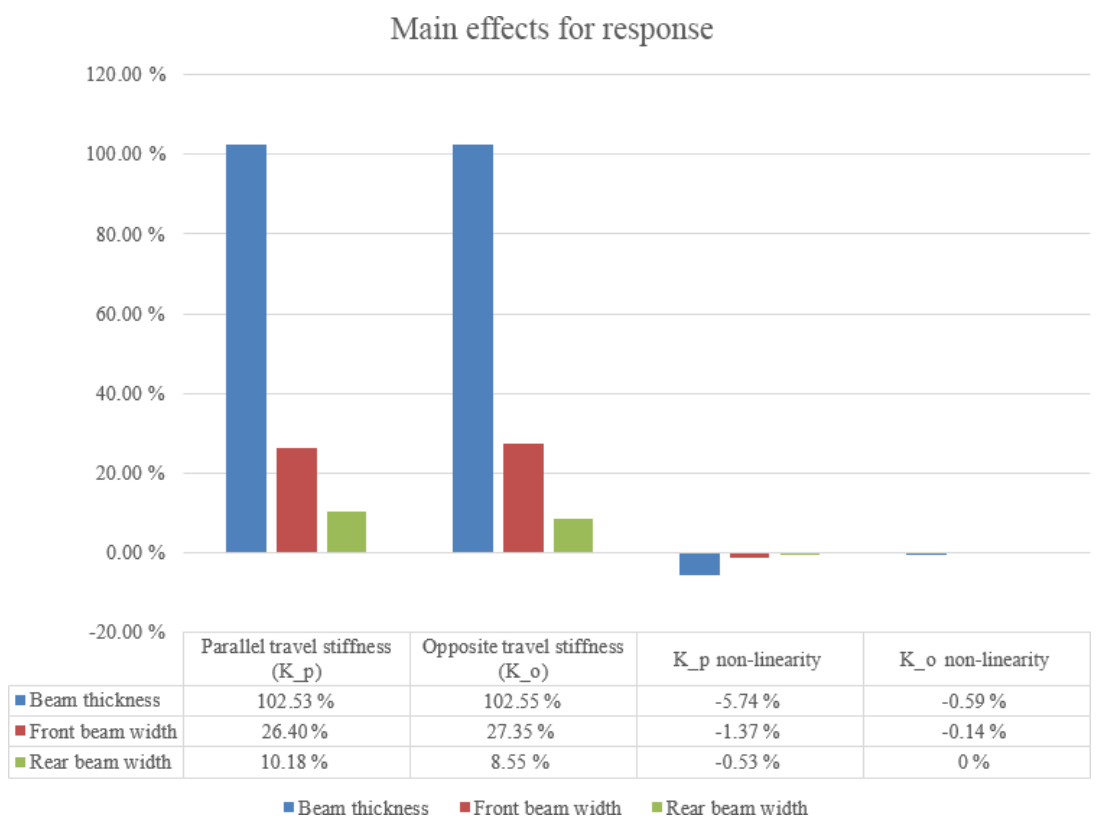

Figure 7. Effect for response.

\subsection{DoE simulation for suspension system}

The beam suspension system is designed using DoE with respect to the reference suspension model (conventional McPherson and SLA) performance target. The stiffness for front/rear beam spring are designed considering the first resonance frequency of the vehicle and vehicle designed load. The anti-roll stiffness, which is known as opposite travel stiffness in this paper is designed with respect to minimize the maximum rolling angle under maximum lateral acceleration and rolling resonance during dynamic maneuver.

During the DoE simulation, the beam stiffness in front suspension for both front and rear beam component in the model are bounded together to have the same value. In the manufacturing phase, the beam for each suspension subsystem (front/rear suspension) should be cut and formed with a single piece of stacked carbon fiber laminate.

The parameter variables have been set within the range considering the available space inside the motor compartment using uniform distribution.

- Beam thickness: $10 \mathrm{~mm} \pm 2 \mathrm{~mm}$

- Front beam width: $90 \mathrm{~mm} \pm 20 \mathrm{~mm}$

- Rear beam width: $40 \mathrm{~mm} \pm 10 \mathrm{~mm}$

The simulation has been performed with Latin Hyper cubic method with 200 iterations, calculating time: $4 \mathrm{~h}$. The effects for response is shown in Fig. 7.

"Beam thickness" influence is most significant as shown in Fig. 7, "beam width" is less significant for its smaller contribution in moment of inertia under suspension-loaded condition, and wider beam cannot be used in the application con- sidering the available space. The "parallel wheel travel nonlinearity" shows an inverse correlation with the beam thickness, the thicker the beam, the non-linearity decreases in the beam spring, which means the spring stiffness is more constant over the whole deformation range (for spring stiffness). It is very interesting to point out that the beam thickness has negligible effect on the opposite travel stiffness linearity (linearity of anti-roll stiffness), it is not clear if the mounting structure used on the beam is the cause of this phenomenon. Unfortunately, it is not possible to investigate more on this phenomenon in MBD solution, which is the main concern of this article.

In the end, the front suspension beam is designed as $100 \mathrm{~mm}$ wide on the front, $40 \mathrm{~mm}$ wide on the rear and $9.75 \mathrm{~mm}$ thick with a parallel/opposite stiffness nonlinearity of 1.151/1.048. It is the best compromise between manufacturing cost and stiffness requirements. The stiffness comparison is available in Fig. 8.

\subsection{Suspension sub-system kinematic simulation}

The kinematic characteristics are focused on the following aspects and the comparison between reference suspension and beam suspension of the simulation results are shown:

- Camber variation

- Toe variation

- Caster

- Beam spring stiffness

- Quasi-static constant radius cornering 

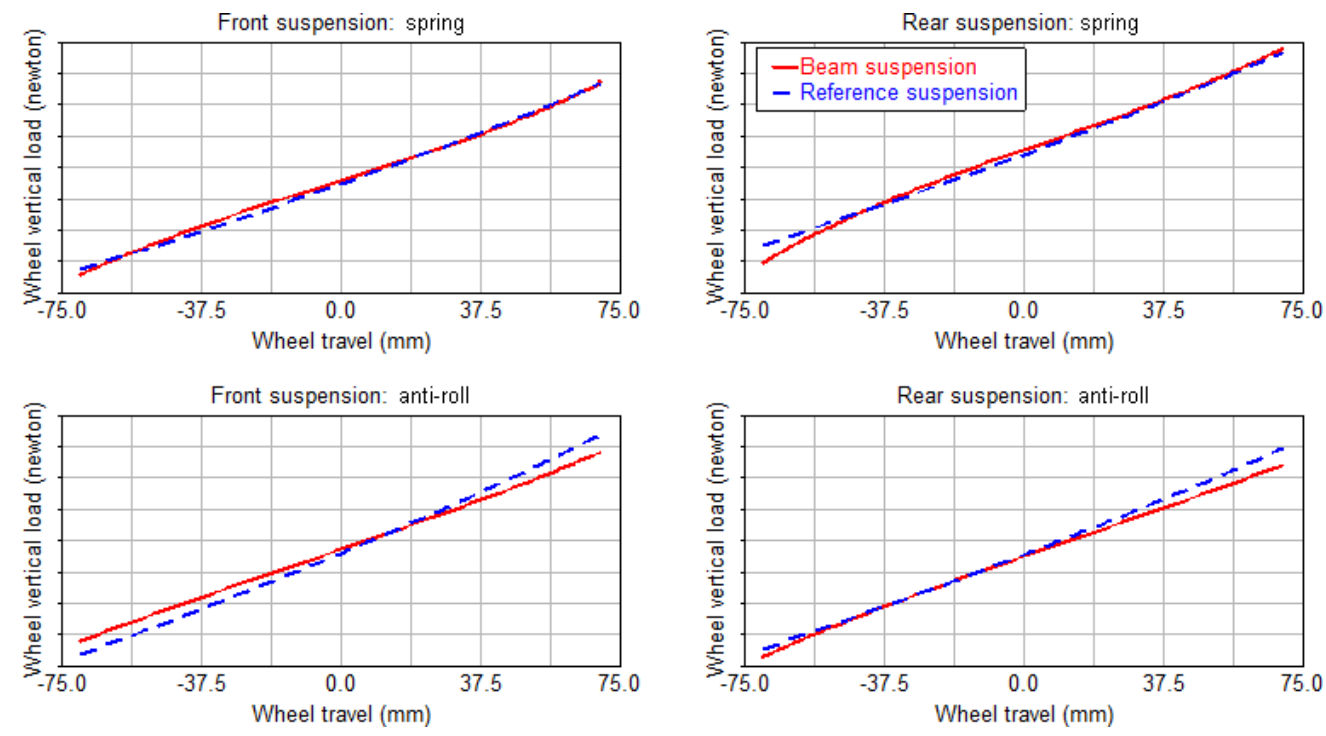

Figure 8. Beam spring stiffness tuning (compression stiffness and anti-roll stiffness).
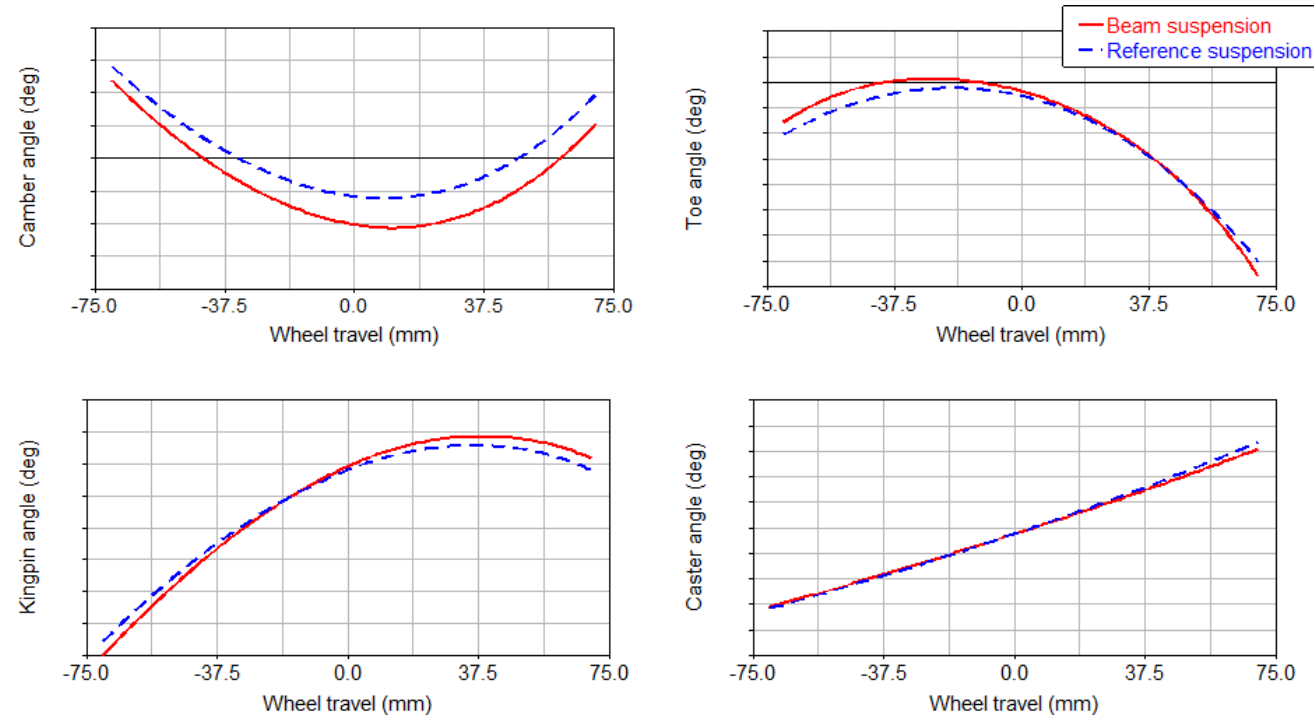

Figure 9. Front suspension kinematics.

Simulations have been done for both reference model and beam model, using the common parallel wheel travel on front and rear suspension. According to the common experience of chassis design for passenger cars (Gunnell, 2008), the expected performance has the following values in Table 2 .

After the reference model has been developed, the beam model is set-up with all the coordinates of hardpoints from reference model, afterwards the tuning of beam model has been done to fit the performance of the reference model. The comparisons of results are shown in the Fig. 9 for the front suspension and Fig. 10 for rear suspension.

As it can be seen, the kinematic characteristics are perfectly reproduced for the beam suspension, such as camber, kingpin, toe-in/out and caster angle variations ${ }^{11}$.

Beam spring stiffness has been carefully tuned with respect to FEM analysis. The correlation for the stiffness has been compromised ${ }^{12}$ for the reason of manufacturing cost.

\footnotetext{
${ }^{11}$ Correlation factor is more than $99 \%$ between the reference curve and beam suspension result, using "Pearson correlation method" (Pearson, 1985)

${ }^{12}$ In order to keep the riding height under different loading condition respect to the reference model, the beam spring stiffness has been set as the primary target, while the anti-roll stiffness is compromised.
} 
Table 2. Performance target* for beam suspension.

\begin{tabular}{llrrr}
\hline Front suspension & Unit & \multicolumn{3}{c}{ Range } \\
\hline Static Camber angle & deg & -0.80 & to & -0.30 \\
Anti-Dive Angle & deg & 5.00 & to & 7.00 \\
Anti-lift Angle & deg & -1.00 & to & -0.20 \\
Ride Steer at $\pm 10 \mathrm{~mm}$ bump & $\operatorname{deg} 100 \mathrm{~mm}^{-1}$ & -1.60 & to & -0.40 \\
Ride Camber at $\pm 10 \mathrm{~mm}$ bump & $\operatorname{deg} 100 \mathrm{~mm}^{-1}$ & -2.00 & to & -1.00 \\
\hline Rear suspension & Unit & & Range & \\
\hline Static Camber Angle & deg & -1.00 & to & 0 \\
Anti-Dive Angle & $\operatorname{deg}$ & 5.00 & to & 7.00 \\
Ride Steer at $\pm 10 \mathrm{~mm}$ bump & $\operatorname{deg} 100 \mathrm{~mm}^{-1}$ & 0.40 & to & 1.60 \\
Ride Camber at $\pm 10 \mathrm{~mm}$ bump & $\operatorname{deg} 100 \mathrm{~mm}^{-1}$ & -3.00 & to & -1.50 \\
\hline
\end{tabular}

* The reference suspension has been designed respect to these requirements.
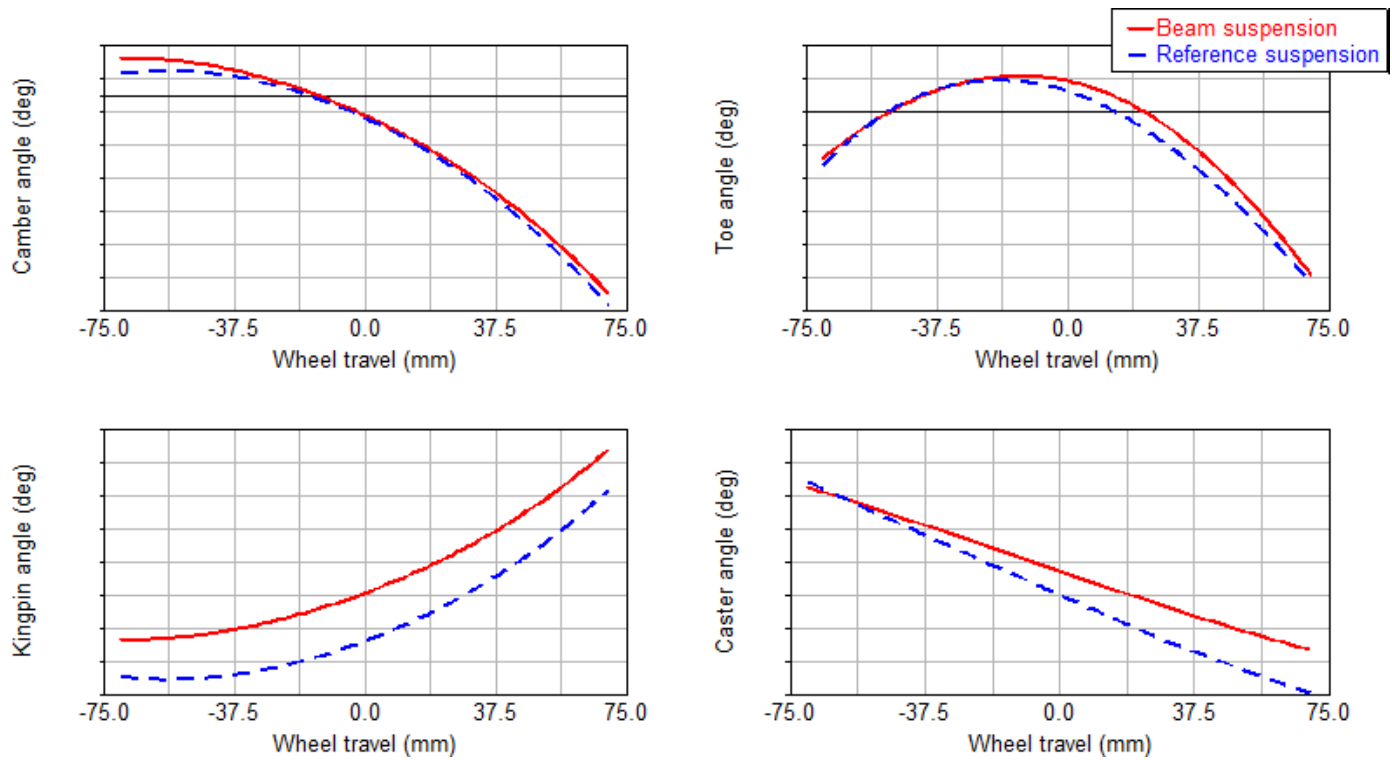

Figure 10. Rear suspension kinematics.
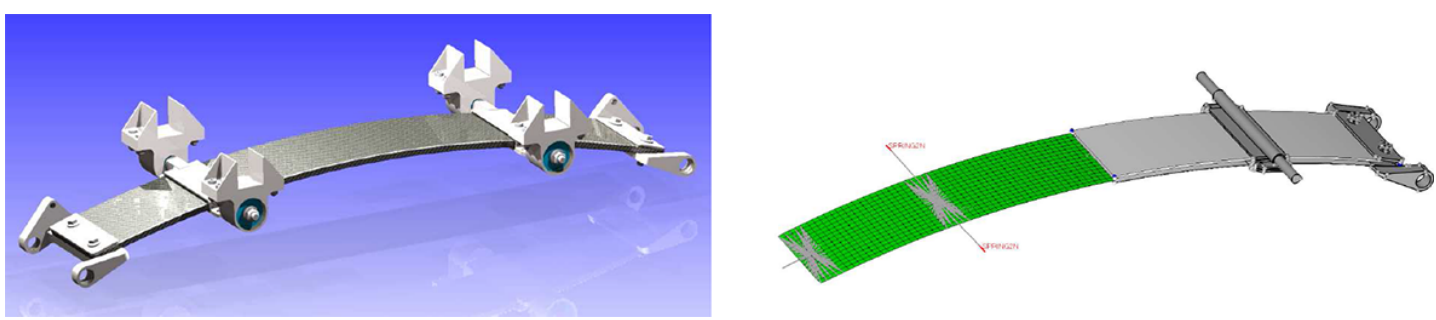

Figure 11. CAD model (left) and FEM model (right), the rear suspension has $\mathrm{H}$ arm layout and the mounting points are located near the beam geometry centre using bushings. 
Table 3. Swept-sine steering simulation result comparison.

\begin{tabular}{llrr}
\hline Yaw rate - Steering angle $^{\mathrm{a}}$ & Unit & Reference model & Beam model \\
\hline Steady Gain & $\mathrm{s}^{-1}$ & 0.40 & 0.43 \\
Gain at $0.5 \mathrm{~Hz}$ & $\mathrm{~s}^{-1}$ & 0.43 & 0.52 \\
Delay at $0.5 \mathrm{~Hz}$ & $\mathrm{~s}$ & 0.029 & 0.029 \\
DFb & - & 1.20 & 1.23 \\
\hline Roll angle - Lateral acceleration & Unit & Reference model & Beam model \\
\hline Steady Gain & deg g & 4.01 & 4.76 \\
Gain at $0.5 \mathrm{~Hz}$ & deg g $^{-1}$ & 4.06 & 4.06 \\
DF & - & 1.74 & 1.05 \\
\hline Slide slip angle - Steering angle & Unit & Reference model & Beam model \\
\hline Steady Gain & - & 0.044 & 0.064 \\
Gain at $0.5 \mathrm{~Hz}$ & - & 0.047 & 0.073 \\
Delay at $0.5 \mathrm{~Hz}$ & $\mathrm{~s}$ & 0.81 & 0.80 \\
DF & - & 1.07 & 1.03 \\
\hline
\end{tabular}

a INPUT - OUTPUT; ${ }^{\mathrm{b}}$ Damping factor $=$ Gain $(\max ) /$ Gain(steady).

The beam has been designed to have uniform thickness. Complex lamination will increase the complexity and uncertainty during manufacturing.

The rear suspension has been designed using the "H arm", the toe angle cannot be efficiently tuned as shown in Fig. 10.

The skid pad simulation ${ }^{13}$ showed a good correlation for under-steering ratio, $37.19 \mathrm{deg} \mathrm{g}^{-1}$ for reference model and $36.26 \mathrm{deg} \mathrm{g}^{-1}$ for beam model.

\subsection{Dynamic simulation-swept sine steering}

The swept sine steering is very important for understanding the full vehicle steering response on frequency domain (Fisher, 2011). The initial speed for the vehicle has been chosen as $80 \mathrm{~km} \mathrm{~h}^{-1}$ and simulation has been performed covering the frequency range 0.05 to $5 \mathrm{~Hz}$. Some representative results are shown in Table 3, the correlation between the beam suspension and reference suspension is good.

At this moment, it is clear that the beam spring suspension proposed can reproduce with a good agreement the performance of the reference suspension (conventional type) in terms of global handling. Which means it is possible to substitute the conventional suspension (coil spring and metallic control arms) with CFRP beam spring suspension for standard passenger vehicles without changing the handling performance of the existing ones.

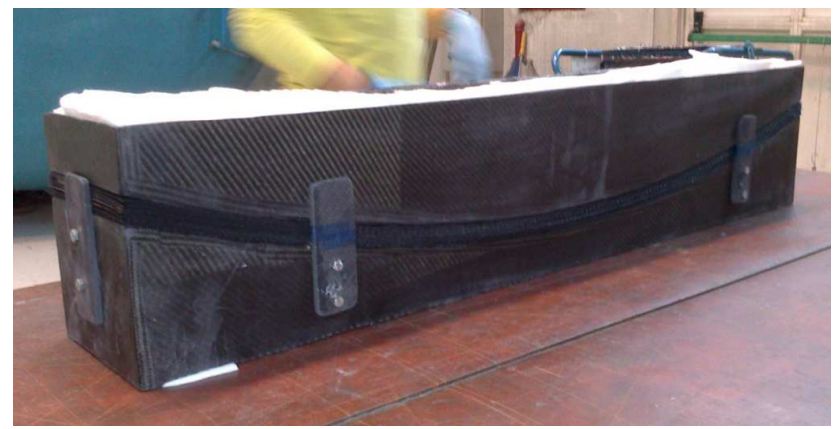

Figure 12. Closed mould filled with Carbon fibre ply and resin.

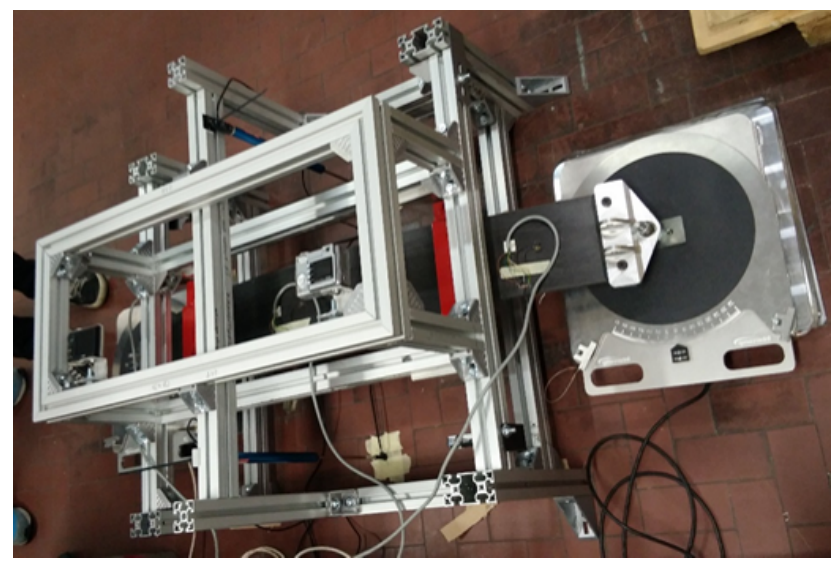

Figure 13. CFRP beam stiffness test bench.

\footnotetext{
${ }^{13}$ Skid pad test with a radius of $40 \mathrm{~m}$ and simulate the vehicle from steady until reaching $1 \mathrm{~g}$ lateral acceleration.
} 
Table 4. Improvements respect to conventional aluminum made suspension system.

\begin{tabular}{llrr}
\hline Improvements & Unit & Value & Difference \\
\hline Front suspension weight reduction & $\mathrm{kg}$ & -10.2 & $-29.1 \%$ \\
Rear suspension weight reduction & $\mathrm{kg}$ & -10.7 & $-26.8 \%$ \\
Wheel to strut distance at front suspension & $\mathrm{mm}$ & -17 & $-34 \%$ \\
Wheel to strut distance at rear suspension & $\mathrm{mm}$ & -22 & $-44 \%$ \\
Strut mounting height at front suspension & $\mathrm{mm}$ & -11 & $-7.4 \%$ \\
Front spring seat plate & - & Removed, no spring needed & $-100 \%$ \\
Rear spring seat plate & - & Removed, no spring needed & $-100 \%$ \\
\hline
\end{tabular}

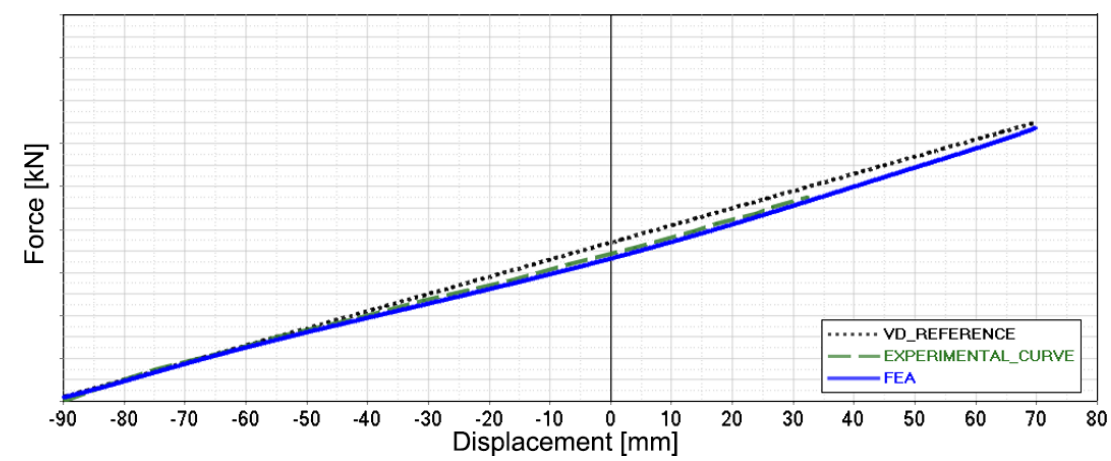

Figure 14. Comparison between FEM analysis result, test bench experimental data and Vehicle Dynamic design target.

\section{Building the Beam suspension}

Using the approved vehicle-dynamics simulation results, the characteristics of the beam components were approximately defined and the first component CAD was created.

FEM analysis has been done to obtain the optimum ply sequence (55 layers) and orientation (Fig. 11).

The beam has been produced completely manually, by using a special vacuum bag technology and autoclave process (Uddin, 2013) to ensure the correct polymerization without any defects in the melt resin. The mold is floating with the carbon fiber to give the better surface finishing and ensure the uniform pressure on the whole piece (shown in Fig. 12).

After cleaning the flanges and drilling for addition components (joint housing), the component is mounted on the chassis prototype.

A simple test bench for evaluating the beam spring stiffness has been made (Fig. 13), with five strain gauges mounted on the spring and weight loaded on the bushing mountings. In Fig. 14, the final Force-Displacement correlation for the beam spring is shown. The displacement starts from $-90 \mathrm{~mm}$ to generate correct preload at normal loaded position. The wheel travel will be limited with the reboundstop integrated in the shock absorber, in order to guarantee the negative wheel travel won't exceed the lower limit $(-70 \mathrm{~mm})$.

The experimental data shows that the beam spring has successfully manufactured with respect to the design target for the beam suspension model.

\section{Conclusion}

In this research, an innovative construction of suspension system has been developed. Using the CFRP beam, the function of lower control arm, coil spring and anti-roll bar has been integrated into a single lightweight composite component. The solution provides new possibilities for saving space and weight reduction (comparison data with conventional vehicle on the market is presented in Table 4).

For front suspension tower, the spring plate has been removed since there is no coil spring present, which benefits the frontal vehicle part design in styling smoother curved surface. When using McPherson suspension on the front, the coil spring is usually placed over the tire to make the strut close to the wheel (decrease scrub radius while keep the kingpin more vertical), the mounting point at suspension tower is very high to fit the coil spring preloaded length. Using CFRP beam suspension can completely solve this issue, the suspension tower can be as low as the shock absorber stroke with additional space for bump stop, the mounting point is lowered more than $10 \mathrm{~mm}$ in this case.

Weight reduction is the main benefit for using CFRP beam suspension. More than $25 \%$ weight is reduced ${ }^{14}$ compared to the conventional suspension arm, coil spring and antiroll bar with their mountings.

\footnotetext{
${ }^{14}$ The suspension beam with mounting weighs less than $5 \mathrm{~kg}$ for one axle.
} 
MBD model has been developed with reference to the performance target. FEM analysis using exact material property was done to predict the component behavior and to design the CAD details for production.

For the future development, the study will be focused on the riding comfort, considering the vibration filtering, the noise proofing and impact smoothing while driving on uneven surface conditions. Also complex lamination design will be studied to improve the correlation with respect to the conventional suspension. Modeling compliant modules or joints (Li and Hao, 2017) in the mounting system for beam spring will be furtherly studied.

The DoE method provide an effective way to find the aspect that is more significantly changing the behavior of the beam spring. The phenomenon of beam thickness effecting non-linearity of anti-roll stiffness should be studied further more.

Production process design will be done later on considering mass production method like RTM and compression molding.

\section{Data availability}

For the reason of this project is subordinate to Fiat Chrysler Automobiles Group, all the detailed data hidden in graphs are confidential and not for public access.

Author contributions. Massimiliana Carello carried out the CFRP beam concept and designed the suspension. Andrea Airale performed the composite material characterization, analysis and production process study. Shuang Xu made the MBD model and completed the suspension fine-tuning. Alessandro Ferraris is in charge of DoE model setting and results analysis.

Competing interests. The authors declare that they have no conflict of interest.

Acknowledgements. This work was supported by the laboratory of Innovative Electric and Hybrid Vehicle DIMEAS - Politecnico di Torino cooperating with BEOND Spin Off (http://www.beondrive.it) and FCA Group.

Edited by: G. Hao

Reviewed by: two anonymous referees

\section{References}

Beardmore, P.: Composite structures for automobiles, Compos. Struct., 5, 163-176, 1986.

Carello, M., Filippo, N., and D'Ippolito, R.: Performance optimization for the XAM hybrid electric vehicle prototype, SAE International Conference, 24-26 April 2012, doi:10.4271/2012-010773, Detroit, 2012.

Carello, M., Airale, A., Ferraris, A., and Messana, A.: XAM 2.0: from student competition to professional challenge, ComputerAided Design and Applications, Taylor \& Francis, 11, 61-67, doi:10.1080/16864360.2014.914412, 2014.

European Aluminum Association: The Aluminum Automotive Manual: Application - Car body - Body structures, ${ }^{\circ}$ European Aluminium Av. de Broqueville, Brussels, 2013.

Fisher, D.: Handbook of driving simulation for engineering, medicine, and psychology, CRC Press, Boca Raton, 2011.

Fuganti, A. and Cupitò, G.: Thixoforming of aluminum alloy for weight saving of a suspension steering knuckle, Metallurgical science and technology, Vol. 18, available at: http://www. gruppofrattura.it/ors/index.php/MST/article/viewFile/1058/1011 (last access: 20 February 2017), 2000.

Gunnell, J.: Standard catalog of Ford, 4th Edn., KP, Iola, Wis., 2008.

Hao, G., Yu, J., and Li, H.: A Brief Review on Nonlinear Modelling Methods and Applications of Compliant Mechanisms, Frontiers of Mechanical Engineering, 11, 119-128, 2016.

Heissing, B.: Chassis handbook fundamentals, driving dynamics, components, mechatronics, perspectives, Vieweg Teubner, Wiesbaden, 2011.

Kulshreshtha, A.: Handbook of polymer blends and composites, 1 st Edn., RAPRA Technology, Shrewsbury, 2002.

Li, H. and Hao, G.: Constraint-force-based approach of modelling compliant mechanisms: Principle and application, Precis. Eng., 47, 158-181, 2017.

Milliken, W. and Milliken, D.: Racecar vehicle dynamics, SAE International, Warrendale, PA, USA, 1995.

Pearson, K.: Notes on regression and inheritance in the case of two parents, P. Roy. Soc. Lond., 58, 240-242, 1895.

Uddin, N.: Developments in fiber-reinforced polymer (FRP) composites for civil engineering, Woodhead Publishing Limited, Cambridge, UK, 2013.

Wood, K.: Composite leaf springs: Saving weight in production suspension systems: Composites World, available at: http://www.compositesworld.com/articles/composite-leafsprings-saving-weight-in-production-suspension-systems (last access: February 2017), 2014.

Yu, W. J. and Kim, H. C.: Double tapered FRP beam for automotive suspension leaf spring, Compos. Struct., 9, 279-300, 1988. 